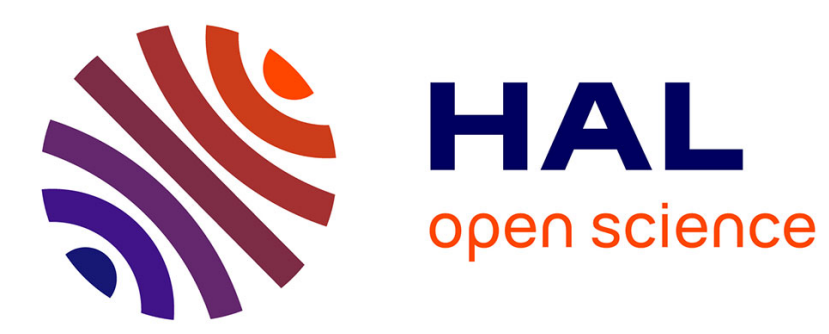

\title{
Spectrum of some integro-differential operators and stability of travelling waves
}

\author{
Arnaud Ducrot, Martine Marion, Vitaly Volpert
}

\section{To cite this version:}

Arnaud Ducrot, Martine Marion, Vitaly Volpert. Spectrum of some integro-differential operators and stability of travelling waves. Nonlinear Analysis: Theory, Methods and Applications, 2011, 74 (13), pp.4455-4473. 10.1016/j.na.2011.04.009 . hal-00653653

\section{HAL Id: hal-00653653 https://hal.science/hal-00653653}

Submitted on 20 Dec 2011

HAL is a multi-disciplinary open access archive for the deposit and dissemination of scientific research documents, whether they are published or not. The documents may come from teaching and research institutions in France or abroad, or from public or private research centers.
L'archive ouverte pluridisciplinaire HAL, est destinée au dépôt et à la diffusion de documents scientifiques de niveau recherche, publiés ou non, émanant des établissements d'enseignement et de recherche français ou étrangers, des laboratoires publics ou privés. 


\title{
Spectrum of Some Integro-Differential Operators and Stability of Travelling Waves
}

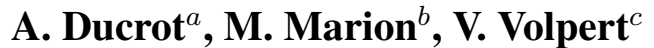 \\ ${ }^{a}$ UMR 5251 CNRS \& INRIA Sud-ouest Anubis, Université Bordeaux, 33000 Bordeaux, France \\ ${ }^{b}$ Université de Lyon, Ecole Centrale de Lyon, UMR 5208 CNRS, 69134 Ecully, France \\ ${ }^{c}$ Institute of mathematics, University Lyon 1, UMR 5208 CNRS \\ 69622 Villeurbanne, France
}

\begin{abstract}
Spectral properties of some integro-differential operators on $\mathbb{R}^{1}$ are studied. Characterization of the principal eigenvalue is obtained in terms of the positive eigenfunction. These results are used to prove local and global stability of travelling waves and to find their speed.
\end{abstract}

Key words: Spectral properties, nonlocal elliptic operator, travelling waves, stability. AMS subject classification: 34K08, 35K57

\section{Introduction}

In this paper we shall study some spectral properties of the linear integro-differential operator

$$
L u=u^{\prime \prime}+c u^{\prime}+a(x) \phi * u+b(x) u,
$$

acting from the Banach space $E=C_{0}^{2}(\mathbb{R})$ into the Banach space $F=C_{0}(\mathbb{R})$. Here $C_{0}(\mathbb{R})$ denotes the space of continuous functions from $\mathbb{R}$ into itself that tend to zero at $x= \pm \infty$ while $E$ is the space of functions $u$ of class $C^{2}$ such that $u, u^{\prime}$ and $u^{\prime \prime}$ belong to $F$. The functions $x \rightarrow a(x)$ and $x \rightarrow b(x)$ are supposed to be bounded and continuous with the limits $a^{ \pm}$and $b^{ \pm}$when $x \rightarrow \pm \infty$, respectively, while $\phi * u$ denotes the convolution product,

$$
\phi * u(x)=\int_{-\infty}^{\infty} \phi(x-y) u(y) d y \quad x \in \mathbb{R}
$$

where $\phi(x)$ is some integrable function. 
The spectrum of the operator $L$ consists of its essential part and of eigenvalues. Here and in the sequel, the essential spectrum is understood as the Fredholm spectrum, namely the set of complex $\lambda$ for which the operator $L-\lambda$ does not satisfy the Fredholm property.

On the one hand, the location of the essential spectrum is an important point when dealing with some nonlinear problems. Indeed, most of the tools of nonlinear analysis, such as the implicit function theorem, bifurcation analysis or topological degree, are based on the Fredholm property and on the related to it classical solvability conditions. Coming back to operator, the location of its essential spectrum is related to the so-called limiting operators

$$
L^{ \pm} u=u^{\prime \prime}+c u^{\prime}+a^{ \pm} \phi * u+b^{ \pm} u,
$$

which are obtained from the operator $L$ if we replace the coefficients $a(x)$ and $b(x)$ by their limits at infinity. The notion of limiting operators is well known in the context of elliptic operators. They were introduced for elliptic problems in general unbounded domains in [19] where the assumption that the coefficient have limits at infinity or that the domain has a special structure were not employed. In the particular situation considered in this work, the definition of the limiting operator is simple and rather explicit. The relationship between the limiting operators and the Fredholm spectrum was elucidated in [2,3]. It was shown that the operators $L^{ \pm}$determine the set of $\lambda \in \mathbb{C}$ such that the operator $L-\lambda$ is normally solvable, which is equivalent to the closeness of its image with a finite dimensional kernel. Under some additional conditions, the codimension of the image is also finite, leading to the Fredholm property. Hence we know the structure of the essential spectrum $\sigma_{e s s}(L)$ of operator $L$.

On the other hand, similarly to elliptic operators in unbounded domains (see [20]), the spectrum of the operator $L$ lies in some left-half plane. The Fredholm property ensures that the set of eigenvalues cannot have accumulation points in some finite part of the complex plane outside of the essential spectrum. Therefore, for any $\sigma_{0}>\sup \operatorname{Re} \sigma_{\text {ess }}(L)$, there exists only a finite number of eigenvalues of the operator $L$ in the half-plane $\operatorname{Re} \lambda \geq \sigma_{0}$. Such a distribution of the eigenvalues of the operator $L$ allows us to define the principal eigenvalue, that is the eigenvalue with the maximal real part. It plays an important role in the analysis of stability of solutions of nonlinear problems. In the case of scalar second order elliptic problems in bounded domains, the Krein-Rutman type theorems affirm that the principal eigenvalue is real, simple and the corresponding eigenfunction is positive. These results use the cone preserving property as well as the compactness of the inverse Laplace operator. The situation becomes more complex when dealing with unbounded domains because of the lack of compactness for the resolvent of the Laplace operator. However, this PerronFrobenius like property of the principal eigenvalue remains valid (we refer to [17]).

The aim of this work is to study the properties of the principal eigenvalue of the integrodifferential operator $L$ when functions $a(x)$ and $\phi(x)$ are non-negative. Then the corresponding evolution equations satisfy the maximum and comparison principles. They will allow us to prove that the principal eigenvalue of the operator is characterized by positiveness of the corresponding eigenfunction. This is the main result of the work (see Theorem 8). Note that such a property does not hold true without the conditions on the functions $a$ and $\phi$. Indeed, otherwise nonmonotone waves may exist and be numerically stable (see for instance $[1,4,9,11]$ ) signifying that the eigenfunction corresponding to the principal eigenvalue, which is the first derivative of the wave, is not 
positive.

These spectral properties have strong implications in nonlinear analysis and more particularly when looking at the stability of monotone travelling wave for some nonlocal parabolic problem of the form

$$
\frac{\partial u(x, t)}{\partial t}=\frac{\partial^{2} u(x, t)}{\partial x^{2}}+f(u(x, t), J(u)(x, t)), \quad t>0, x \in \mathbb{R}
$$

wherein we have set

$$
J(u)(x, t)=\int_{\mathbb{R}} \phi(x-y) u(y, t) d y,
$$

and where $f=f(u, v)$ is some regular map. This kind of equations arises in many applications (see for instance $[6,14,15]$ and the references cited therein).

Let us recall that a travelling wave for (1.2) is particular solution of the form $u(x, t)=w(x-c t)$ where $c \in \mathbb{R}$ is the wave speed, so that $w$ is a stationary solution of the problem

$$
\frac{\partial u(x, t)}{\partial t}=\frac{\partial^{2} u(x, t)}{\partial x^{2}}+c \frac{\partial u(x, t)}{\partial x}+f(u(x, t), J(u)(x, t)), \quad t>0, x \in \mathbb{R} .
$$

The linear stability of $w$ is related to the spectral properties of operator $L$ with

$$
a(x)=\frac{\partial f(w(x), J(w)(x))}{\partial v}, \quad b(x)=\frac{\partial f(w(x), J(w)(x))}{\partial u} .
$$

If we assume that the map $f: \mathbb{R}^{2} \rightarrow \mathbb{R}$ is of the class $C^{1}$ and satisfies

$$
\frac{\partial f(u, v)}{\partial v} \geq 0, \quad \forall(u, v) \in \mathbb{R}^{2}
$$

we obtain, as a consequence of the spectral theory developed in this work, that the wave solution $w$ is globally stability with shift in a scale of weighted spaces depending on the asymptotic behaviour at $x= \pm \infty$ of the initial data as well as on the location of the essential spectrum of operator $L$. This kind of result is well known for reaction-diffusion equations (see [18] and the references therein). A result concerning the stability of waves for (1.2) was proved by Chen in [5] by using upper and lower-solution method, also well known for the scalar reaction-diffusion equations (see [7]). The advantage of the spectral method is that it provides a more precise stability result depending on the properties of the initial data. It also provides a minimax representation of the wave speed. One may also notice that this method applies in the multidimensional situation and has rather simple extension to systems of equations. This point will be the aim of a forthcoming work. Let us mention that these results may also apply to reaction-diffusion equations with delay.

The paper is organized as follow. In Section 2 we prove some comparison results. Section 3 investigates the location of the spectrum of operator $L$. Section 4 derives some consequences for the local stability of waves for problem (1.2) while Section 5 provides global stability result and the minimax representation of the wave speed. Finally Section 6 gives some examples of application in the context of population dynamics and chemical kinetics. 


\section{Positiveness and comparison theorems}

\subsection{Existence of solutions}

Consider the equation involving a scalar function $u=u(x, t), x \in \mathbb{R}, t \geq 0$

$$
\frac{\partial u}{\partial t}=\frac{\partial^{2} u}{\partial x^{2}}+f(u, J(u))
$$

together with the initial condition

$$
u(x, 0)=u_{0}(x), x \in \mathbb{R} .
$$

Here the function $f$ is of class $C^{1}$ from $\mathbb{R}^{2}$ into $\mathbb{R}$ with

$$
|f(u, v)| \leq \kappa(1+|u|+|v|), \forall(u, v) \in \mathbb{R}^{2},
$$

where herefater $\kappa$ denotes some positive constant. The term $J(v)$ acts on a bounded function $v=v(y), y \in \mathbb{R}$ and is defined by the convolution product

$$
J(v)(x)=\phi * v(x)=\int_{-\infty}^{\infty} \phi(x-y) v(y) d y, \quad \forall x \in \mathbb{R}
$$

where $\phi(x)$ is a given integrable function.

We first investigate the existence of solutions of this problem.

Theorem 1. Under the above assumptions, let $u_{0} \in L^{\infty}(\mathbb{R})$. Then there exists a unique solution $u$ of the Cauchy problem (2.1)-(2.2) that satisfies $u \in L^{\infty}(\mathbb{R} \times[0, T])$ for all $T>0$. The function $u$ is continuous with respect to both variables $(x, t)$ on $\mathbb{R} \times[0,+\infty[$ and possesses a continuous second derivative with respect to $x \in \mathbb{R}$ and first derivative with respect to t on $\mathbb{R} \times] 0,+\infty[$.

Proof. Let us denote by $\|.\|_{\infty}$ the supremum norm on $L^{\infty}(\mathbb{R})$. For $v \in L^{\infty}(\mathbb{R})$ we set

$$
w(x)=f\left(v(x), \int_{-\infty}^{\infty} \phi(x-y) v(y) d y\right) .
$$

Clearly $w \in L^{\infty}(\mathbb{R})$ and let $F$ denote the mapping $v \rightarrow w$.

Lemma 2. The mapping $F$ is Lipschitz continuous on the bounded sets of $L^{\infty}(\mathbb{R})$. Furthermore there exists a constant $\kappa$ such that $\|F(v)\|_{\infty} \leq \kappa\|v\|_{\infty}$ for all $v \in L^{\infty}(\mathbb{R})$.

Next let $S(t)$ denote the semi-group associated to the heat equation. It is well known that the following estimate holds :

$$
\left\|S(t) u_{0}\right\|_{\infty} \leq\left\|u_{0}\right\|_{\infty}, \forall t>0 \text { and } u_{0} \in L^{\infty}(\mathbb{R}) .
$$

For $u=u(x, t)$, let $u(t)$ be the function $x \rightarrow u(x, t)$. Then the solution $u$ of $(2.1),(2.2)$ reads 


$$
u(t)=S(t) u_{0}+\int_{0}^{t} S(t-s) F(u(s)) d s .
$$

By looking for fixed points of the operator given by the right hand-side of (2.5) in the space $L^{\infty}(\mathbb{R} \times[0, T])$ with $T>0$, we easily conclude that such a fixed point exists first for sufficiently small $T$ and then for all $T>0$. This provides the existence and uniqueness result in Theorem 1 .

Corollary 3. Under the assumptions of Theorem 1, assume furthermore that the initial data possesses limits at infinity

$$
u_{ \pm}^{0}=\lim _{x \rightarrow \pm \infty} u_{0}(x)
$$

Then the following limits

$$
u_{ \pm}(t)=\lim _{x \rightarrow \pm \infty} u(x, t)
$$

also exist and satisfy the equation

$$
\frac{d u_{ \pm}(t)}{d t}=f\left(u_{ \pm}(t),\left(\int_{\mathbb{R}} \phi\right) u_{ \pm}(t)\right)
$$

Proof. It consists in taking the limits $x \rightarrow \pm \infty$ in the iterative procedure in the proof of Theorem 1. For example we have

$$
S(t) u_{0} \rightarrow u_{ \pm}^{0}, \int_{0}^{t} S(t-s) F\left(S(s) u_{0}\right) d s \rightarrow \int_{0}^{t}\left[f\left(u_{ \pm}^{0},\left(\int_{-\infty}^{\infty} \phi\right) u_{ \pm}^{0}\right)\right] d s .
$$

The details are left to the reader.

\subsection{Maximum and comparison principle}

This section is devoted to some comparison results for (2.1)-(2.2) as well as for some corresponding linear equations.

Let us introduce the operator

$$
\mathcal{L}[u]=\frac{\partial^{2} u}{\partial x^{2}}+c \frac{\partial u}{\partial x}+f(u, J(u))-\frac{\partial u}{\partial t},
$$

where $c \in \mathbb{R}$ is given. In addition to (2.3) we will assume that

$$
\begin{gathered}
\operatorname{supp} \phi \subset[-N, N], \text { for some given } N>0, \quad \phi(x) \geq 0, \\
\frac{\partial f(u, v)}{\partial v} \geq 0, \quad \forall(u, v) \in \mathbb{R}^{2} .
\end{gathered}
$$

Then the following comparison result holds : 
Theorem 4. Under assumptions (2.3), (2.8) and (2.9), let $T>0$ be given and let $u$ and $v$ be two functions in $C^{0}(\mathbb{R} \times[0, T]) \cap C^{2,1}(\mathbb{R} \times(0, T]) \cap L^{\infty}(\mathbb{R} \times[0, T])$ satisfying

$$
\begin{aligned}
& u(x, 0) \leq v(x, 0), \quad \forall x \in \mathbb{R}, \\
& \mathcal{L}[u](x, t) \geq \mathcal{L}[v](x, t) \quad \forall t \in(0, T), \forall x \in \mathbb{R} .
\end{aligned}
$$

Then we have $u(x, t) \leq v(x, t)$ for any $x \in \mathbb{R}$ and $t \in[0, T]$.

In order to prove this comparison result, we first investigate some associated linear equation. For that purpose we are given $T>0$ and three functions $a \equiv a(x, t), b \equiv b(x, t)$ and $c \equiv c(x, t)$ defined on $\mathbb{R} \times[0, T]$. We consider the operator

$$
L[u]=\frac{\partial^{2} u}{\partial x^{2}}+c(x, t) \frac{\partial u}{\partial x}+a(x, t) J(u)+b(x, t) u-\frac{\partial u}{\partial t} .
$$

We will assume that

$$
\begin{aligned}
& \text { The functions } a, b \text { and } c \text { are continuous and bounded on } \mathbb{R} \times[0, T] \text {, } \\
& a(x, t) \geq 0, \quad \forall t \in(0, T), \forall x \in \mathbb{R} \text {. }
\end{aligned}
$$

Then the following positiveness result holds:

Theorem 5. Let assumption (2.12) be satisfied. Let $u \in C^{0}(\mathbb{R} \times[0, T]) \bigcap C^{2,1}(\mathbb{R} \times(0, T])$ satisfying

$$
\begin{aligned}
& L[u](x, t) \leq 0, \quad \forall t \in(0, T], \forall x \in \mathbb{R}, \\
& u(x, 0) \geq 0, \quad \forall x \in \mathbb{R}, \\
& u(x, t) \geq-B, \quad \forall t \in[0, T], \forall x \in \mathbb{R},
\end{aligned}
$$

for some constant $B>0$. Then we have $u(x, t) \geq 0$ for any $(x, t) \in \mathbb{R} \times[0, T]$.

Furthermore the following alternative holds : either $u(x, t) \equiv 0$ for any $(x, t) \in \mathbb{R} \times[0, T]$ or $u(x, t)>0$ for any $(x, t) \in \mathbb{R} \times(0, T]$.

Proof. Let us consider the function

$$
v(x, t)=u(x, t) \exp \left(-\sqrt{1+x^{2}}-\nu t\right)
$$

defined for $x \in \mathbb{R}$ and $0 \leq t \leq T$ where $\nu$ is some positive constant to be specified later. Obviously, we have :

$$
v(x, t) \geq-B \exp \left(-\sqrt{1+x^{2}}\right) .
$$

Therefore the following inequality holds

$$
\liminf _{|x| \rightarrow+\infty} v(x, t) \geq 0
$$


uniformly with respect to $t \in[0, T]$ and $\nu \geq 0$. For convenience let us set

$$
\theta(x)=\sqrt{1+x^{2}} \text { and } h(x, t)=\exp (\theta(x)+\nu t) .
$$

It follows easily from (2.13) and (2.14) that the function $v$ satisfies the differential inequality

$$
\bar{L}[v]=\frac{\partial^{2} v}{\partial x^{2}}+\bar{c} \frac{\partial v}{\partial x}+\frac{a}{h} J(v h)+\bar{b} v-\frac{\partial v}{\partial t} \leq 0
$$

where

$$
\begin{aligned}
& \bar{c}(x, t)=c(x, t)+2 \theta^{\prime}(x), \\
& \bar{b}(x, t)=b(x, t)+\theta^{\prime \prime}(x)+\theta^{\prime}(x)^{2}+\theta^{\prime}(x) c(x, t)-\nu .
\end{aligned}
$$

We claim that we can choose $\nu$ sufficiently large so that

$$
\bar{L}[1]=\frac{a}{h} J(h)+\bar{b}<0 \text { on } \mathbb{R} \times[0, T]
$$

Indeed, in view of the estimate

$$
\frac{a(x, t)}{h(x, t)} J(h)(x, t) \leq a(x, t)\|\phi\|_{\infty} \int_{-N}^{N} \exp (\theta(x-y)-\theta(x)) d y
$$

the function $\frac{a}{h} J(h)$ is bounded on $\mathbb{R} \times[0, T]$. Since $a, b, c, \theta^{\prime}$ and $\theta^{\prime \prime}$ are also bounded the above property easily follows.

Next, for $\epsilon>0$, the function $w=v+\epsilon$ satisfies

$$
\liminf _{|x| \rightarrow+\infty} w(x, t) \geq \epsilon
$$

uniformly with respect to $t \in[0, T]$. Therefore there exists $R>0$ such that

$$
w(x, t)>0, \text { for }|x| \geq R \text { and } 0 \leq t \leq T .
$$

We aim to derive the positivity of $w$ on $[-R, R] \times[0, T]$. Note that, thanks to (2.15) and (2.17), $w$ satisfies the differential inequality

$$
\bar{L}[w]=\bar{L}[v]+\epsilon \bar{L}[1]<0 \text { on } \mathbb{R} \times(0, T] .
$$

Since $w(x, 0)=u(x, 0) \exp \left(-\sqrt{1+x^{2}}\right)+\epsilon>0$ we necessarily have $w(x, t)>0$ on $[-R, R] \times$ $\left[0, t^{*}\right]$ for some $t^{*}>0$. Let $t^{0}$ denotes the supremum of the $t^{*}>0$ having this property. If $t^{0}<T$, the function $w\left(., t^{0}\right)$ is positive and vanishes at some point $x_{0}$. Due to (2.18) we have $\left.x_{0} \in\right]-R, R[$ and

$$
\bar{L}[w]\left(x_{0}, t_{0}\right)=\frac{\partial^{2} w}{\partial x^{2}}\left(x_{0}, t_{0}\right)+\frac{a\left(x_{0}, t_{0}\right)}{h\left(x_{0}, t_{0}\right)} J(w h)\left(x_{0}, t_{0}\right)-\frac{\partial w}{\partial t}\left(x_{0}, t_{0}\right) \geq 0 .
$$

This inequality contradicts (2.19). Hence $t^{0}=T$ and $w \geq 0$ on $[-R, R] \times[0, T]$. 
Thanks to (2.18), we conclude that $w \geq 0$ on $\mathbb{R} \times[0, T]$. Since $\epsilon>0$ is arbitrary the positivity of $w$ yields the one of $v$ by taking the limit $\epsilon \rightarrow 0$. By (2.14), the positivity of $u$ follows readily.

Finally note that $u$ satisfies the differential inequality

$$
\frac{\partial^{2} u}{\partial x^{2}}+c(x, t) \frac{\partial u}{\partial x}+b(x, t) u-\frac{\partial u}{\partial t} \leq-a(x, t) J(u) \leq 0
$$

together with $u(x, 0) \geq 0$. Hence classical properties of parabolic operators yield the alternative in Theorem 5 whose proof is now complete.

We now aim to complete the proof of Theorem 4 .

Proof of Theorem 4. The function $w=v-u$ satisfies

$$
\frac{\partial^{2} w}{\partial x^{2}}+c \frac{\partial w}{\partial x}+f(v, J(v))-f(u, J(u))-\frac{\partial w}{\partial t} \leq 0
$$

together with $w(x, 0) \geq 0$. Then $f(v, J(v))-f(u, J(u))=b(x, t) w+a(x, t) J(w)$ for some bounded functions $a$ and $b$ and $a(x, t) \geq 0$ due to (2.9). Therefore Theorem 5 applies and provides the positivity of $w$.

Next let us investigate the maximum principle for some corresponding elliptic operator. In order to state our result, let us introduce two functions $a \equiv a(x), b \equiv b(x)$, a real number $c \in \mathbb{R}$ and the one-dimensional elliptic operator

$$
L u=u^{\prime \prime}+c u^{\prime}+a(x) \phi * u+b(x) u .
$$

Besides (2.8) let us also assume that

the functions $a$ and $b$ are continuous and bounded on $\mathbb{R}$,

$$
a(x) \geq 0 \quad \forall x \in \mathbb{R}, \quad\left(\int_{\mathbb{R}} \phi(t) d t\right) a(x)+b(x)<0 \quad \forall|x|>N .
$$

Then we have the following elliptic comparison result:

Lemma 6. Let conditions (2.8) and (2.22) be satisfied. Let $u \in C^{2}(\mathbb{R}) \bigcap L^{\infty}(\mathbb{R})$ be a given function such that $u \geq 0$ on $[-N, N], L u \leq 0$ on $\mathbb{R}$ and

$$
\liminf _{|x| \rightarrow \infty} u(x) \geq 0
$$

Then we have $u \geq 0$ on $\mathbb{R}$. 
Proof. Let us assume that there exists $x_{0} \in \mathbb{R}$ with $\left|x_{0}\right|>N$ such that $u\left(x_{0}\right)<0$. In view of (2.23), one may assume that the function $u$ realizes its minimum value at $x_{0}$. Then, for any $y \in \mathbb{R}$, we have $u\left(x_{0}-y\right) \geq u\left(x_{0}\right)$, so that $\phi * u\left(x_{0}\right)-\left(\int_{\mathbb{R}} \phi(t) d t\right) u\left(x_{0}\right) \geq 0$. On the other hand we have $u^{\prime}\left(x_{0}\right)=0, u^{\prime \prime}\left(x_{0}\right) \geq 0$. Thus we get

$$
u^{\prime \prime}\left(x_{0}\right)+\left(b\left(x_{0}\right)+\left(\int_{\mathbb{R}} \phi(t) d t\right) a\left(x_{0}\right)\right) u\left(x_{0}\right)+a\left(x_{0}\right)\left(\phi * u-\left(\int_{\mathbb{R}} \phi(t) d t\right) u\right)\left(x_{0}\right)>0 .
$$

This contradicts $L u\left(x_{0}\right) \leq 0$. Thus $u(x) \geq 0$ for any $x \in \mathbb{R}$ and the result follows.

Lemma 7. Let conditions (2.8) and (2.22) be satisfied. Let $u \in C^{2}(\mathbb{R}) \bigcap L^{\infty}(\mathbb{R})$ satisfy $u \geq 0$ and $L u \leq 0$. Then either $u \equiv 0$ or $u>0$ on $\mathbb{R}$.

Proof. Let us first note that since $u \geq 0$ and $a \geq 0$ one has $a(x) \phi * u(x) \geq 0, \quad \forall x \in \mathbb{R}$. Hence the inequality $L u \leq 0$ implies that

$$
u^{\prime \prime}+c u^{\prime}+b(x) u \leq 0,
$$

and the result follows from classical properties of elliptic operators.

\section{Spectral properties}

The aim of this section is to study some spectral properties of a linear elliptic operator with a nonlocal term. The operator reads

$$
L u=u^{\prime \prime}+c u^{\prime}+a(x) \phi * u+b(x) u,
$$

and it is considered as acting from the Banach space $E=C_{0}^{2}(\mathbb{R})$ into the Banach space $F=$ $C_{0}(\mathbb{R})$. Here $C_{0}(\mathbb{R})$ denotes the space of continuous functions from $\mathbb{R}$ into itself that tend to zero at $x= \pm \infty$ while $E$ is the space of functions $u$ of class $C^{2}$ such that $u, u^{\prime}$ and $u^{\prime \prime}$ belong to $F$.

Here $c \in \mathbb{R}$ is some given constant while $a$ and $b$ are functions from $\mathbb{R}$ into itself satisfying

$$
\begin{aligned}
& a \text { and } b \text { are continuous and bounded on } \mathbb{R}, \\
& \text { the following limits } a^{ \pm}=\lim _{x \rightarrow \pm \infty} a(x), b^{ \pm}=\lim _{x \rightarrow \pm \infty} b(x) \text { exist, } \\
& a^{ \pm}+b^{ \pm}<0, \quad a(x) \geq 0 \quad \forall x \in \mathbb{R} .
\end{aligned}
$$

Also

$$
\phi(x) \geq 0, \quad \forall x \in \mathbb{R}, \quad \operatorname{Supp} \phi \subset[-N, N] \text { for some given } N>0, \int_{-\infty}^{\infty} \phi(x) d x=1 .
$$

In this section we shall assume that the operator $L$ has the zero eigenvalue associated with a positive eigenvector $u_{0}$ and we shall show a Perron-Frobenius like result for $L$. The precise result is the following: 
Theorem 8. Let assumptions (3.2)-(3.3) be satisfied. Furthermore suppose that there exists some $u_{0} \in E$ with $u_{0}>0$ solution of the equation

$$
L u_{0}=0 .
$$

Then zero is a simple eigenvalue of the operator $L$ while all the other eigenvalues lie in the left half-plane $\{z \in \mathbb{C}: \Re z<0\}$.

Remark 9. A similar result also holds true when the operator $L$ is considered as acting on some Sobolev spaces. The proof in that case can be obtained by a slight modification of the one given below.

We start by deriving some asymptotic properties of the eigenvector $u_{0}(x)$ as $x \rightarrow \pm \infty$. For that purpose let us introduce the so-called limiting operators defined by

$$
L^{ \pm} u=u^{\prime \prime}+c u^{\prime}+a^{ \pm} \phi * u+b^{ \pm} u,
$$

where the coefficients $a(x)$ and $b(x)$ have been replaced by their limits at $\pm \infty$. The maps $x \rightarrow$ $\exp \left(-\alpha_{ \pm} x\right)$ are solutions of the equations $L^{ \pm} u=0$ provided that

$$
\begin{aligned}
& \alpha^{+2}-c \alpha^{+}+a^{+} \int_{-\infty}^{\infty} \phi(z) e^{\alpha^{+} z} d z+b^{+}=0 \\
& \alpha^{-2}+c \alpha^{-}+a^{-} \int_{-\infty}^{\infty} \phi(z) e^{-\alpha^{-} z} d z+b^{-}=0 .
\end{aligned}
$$

The existence of such solutions will be guaranteed by the following lemma :

Lemma 10. Under assumption (3.3), let $\delta$ and $\gamma$ be two given real numbers with

$$
\delta \geq 0, \quad \delta+\gamma<0 .
$$

Then the equations

$$
\alpha^{2} \pm c \alpha+\delta \int_{-\infty}^{\infty} \phi(z) e^{\mp \alpha z} d z+\gamma=0
$$

have respectively a unique solution $\alpha^{ \pm}>0$. Moreover we have

$$
0<\alpha^{ \pm} \leq \frac{c+\sqrt{c^{2}+4|\gamma|}}{2}
$$

Proof. We shall only consider the equation

$$
\alpha^{2}-c \alpha+\delta \int_{-\infty}^{\infty} \phi(z) e^{\alpha z} d z+\gamma=0
$$

Let $h:[0, \infty) \rightarrow \mathbb{R}$ be defined by

$$
h(\alpha)=\alpha^{2}-c \alpha+\delta \int_{-\infty}^{\infty} \phi(z) e^{\alpha z} d z+\gamma
$$


In view of (3.3), $h$ is of class $C^{\infty}$ with $h(0)=\delta+\gamma<0$ and $h(\alpha) \geq \gamma-c \alpha+\alpha^{2}$. These properties guarantee the existence of $\alpha^{+}>0$ such that $h\left(\alpha^{+}\right)=0$ and provide the upper bound (3.6). Finally, due to

$$
h^{\prime \prime}(\alpha)=2+\delta \int_{-\infty}^{\infty} z^{2} \phi(z) e^{\alpha z} d z>0
$$

the function $h$ is convex. Consequently, since $h(0)<0$, the equation $h(\alpha)=0$ has a unique positive solution. This completes the proof of the lemma.

Choosing $\delta=a^{ \pm}$and $\gamma=b^{ \pm}$, Lemma 10 applies thanks to assumption (3.2) and provides the existence of the two unique numbers $\alpha^{ \pm}>0$ satisfying (3.4).

We can now state the following result on $u_{0}$.

Lemma 11. Under assumptions (3.2)-(3.3), let $\varepsilon_{0}>0$ such that

$$
\left(a^{ \pm}-\varepsilon_{0}\right)^{+}+b^{ \pm}-\varepsilon_{0}<0 \text {, with }\left(a^{ \pm}-\varepsilon_{0}\right)^{+}=\max \left(a^{ \pm}-\varepsilon_{0}, 0\right) .
$$

Then for each $\varepsilon \in\left(0, \varepsilon_{0}\right)$ there exists a unique pair $\left(\alpha_{\varepsilon}^{+}, \alpha_{\varepsilon}^{-}\right) \in(0, \infty)^{2}$ satisfying the equations

$$
\begin{aligned}
& \alpha_{\varepsilon}^{+2}-c \alpha_{\varepsilon}^{+}+\left(a^{+}-\varepsilon\right)^{+} \int_{-\infty}^{\infty} \phi(z) e^{\alpha_{\varepsilon}^{+} z} d z+b^{+}-\varepsilon=0 \\
& \alpha_{\varepsilon}^{-2}+c \alpha_{\varepsilon}^{-}+\left(a^{-}-\varepsilon\right)^{+} \int_{-\infty}^{\infty} \phi(z) e^{-\alpha_{\varepsilon}^{-} z} d z+b^{-}-\varepsilon=0 .
\end{aligned}
$$

Moreover we have

$$
\lim _{\varepsilon \rightarrow 0^{+}}\left(\alpha_{\varepsilon}^{+}, \alpha_{\varepsilon}^{-}\right)=\left(\alpha^{+}, \alpha^{-}\right),
$$

and for each $\varepsilon \in\left(0, \varepsilon_{0}\right)$ there exists $m_{\varepsilon}>0$ such that

$$
\liminf _{x \rightarrow \infty} e^{\alpha_{\varepsilon}^{+} x} u_{0}(x) \geq m_{\varepsilon}, \quad \liminf _{x \rightarrow-\infty} e^{-\alpha_{\varepsilon}^{-} x} u_{0}(x) \geq m_{\varepsilon} .
$$

Proof. Note that the existence of $\varepsilon_{0}>0$ follows from (3.2). For $\varepsilon \in\left(0, \varepsilon_{0}\right)$, we have $\left(a^{ \pm}-\varepsilon\right)^{+}+$ $b^{ \pm}-\varepsilon<0$ and there exists $N_{\varepsilon}>N$ such that

$$
a(x) \geq\left(a^{+}-\varepsilon\right)^{+}, \quad b(x) \geq b^{+}-\varepsilon \forall x \geq N_{\varepsilon} .
$$

Let $L_{\varepsilon}$ be the operator defined by

$$
L_{\varepsilon} u=u^{\prime \prime}+c u^{\prime}+\left(a^{+}-\varepsilon\right)^{+} \phi * u+\left(b^{+}-\varepsilon\right) u, \quad u \in E .
$$

Since $u_{0}>0$, we have

$$
L_{\varepsilon} u_{0} \leq L u_{0}=0, \quad \forall x \geq N_{\varepsilon} .
$$

Next, Lemma 10 provides the existence of a unique number $\alpha_{\varepsilon}^{+}>0$ such that

$$
\alpha_{\varepsilon}^{+^{2}}-c \alpha_{\varepsilon}^{+}+\left(a^{+}-\varepsilon\right)^{+} \int_{-\infty}^{\infty} \phi(z) e^{\alpha_{\varepsilon}^{+} z} d z+b^{+}-\varepsilon=0 .
$$


The function $v_{\varepsilon}(x)=u_{0}\left(N_{\varepsilon}\right) e^{-\alpha_{\varepsilon}^{+}\left(x-N_{\varepsilon}\right)}$ satisfies the equality $L_{\varepsilon} v_{\varepsilon}=0$ together with $v_{\varepsilon}\left(N_{\varepsilon}\right)=$ $u_{0}\left(N_{\varepsilon}\right)$.

Now since $u_{0}(x)$ is a positive function, there exists $\nu>0$ such that

$$
\nu v_{\varepsilon}(x) \leq u_{0}(x), \quad N_{\varepsilon}-N \leq x \leq N_{\varepsilon} .
$$

We aim to show that

$$
\nu v_{\varepsilon}(x) \leq u_{0}(x), \quad \forall x \geq N_{\varepsilon} .
$$

Let us consider the function $w(x)=u_{0}(x)-\nu v_{\varepsilon}(x)$. It satisfies $L_{\varepsilon} w \leq 0, \forall x \geq N_{\varepsilon}$ together with $w(x) \geq 0, \forall x \in\left[N_{\varepsilon}-N, N_{\varepsilon}\right]$. Suppose that $w(x)<0$ for some $x>N_{\varepsilon}$. Since $\lim _{x \rightarrow \infty} w(x)=0$, the function $w$ attains its minimum on $\left[N_{\varepsilon},+\infty\left[\right.\right.$ at some $x_{0}>N_{\varepsilon}$ and $w\left(x_{0}\right)<0$. Setting $\hat{w}(x)=w(x)-w\left(x_{0}\right)$, we have

$$
L_{\varepsilon} w=L_{\varepsilon} \hat{w}+L_{\varepsilon} w\left(x_{0}\right)=L_{\varepsilon} \hat{w}+\left(\left(a^{+}-\varepsilon\right)^{+}+b^{+}-\varepsilon\right) w\left(x_{0}\right) \leq 0, \quad \forall x>N_{\varepsilon} .
$$

Let us check that the left hand-side of this inequality is strictly positive at $x=x_{0}$. This contradiction will prove (3.12). Indeed, the last expression in the left-hand side is strictly positive. It remains to verify that $L_{\varepsilon} \hat{w}\left(x_{0}\right) \geq 0$. This follows from

$$
\hat{w}^{\prime \prime}\left(x_{0}\right) \geq 0, \quad \hat{w}^{\prime}\left(x_{0}\right)=0, \quad \hat{w}\left(x_{0}\right)=0, \quad \phi * \hat{w}\left(x_{0}\right)=\int_{-N}^{N} \phi(y) \hat{w}\left(x_{0}-y\right) d y \geq 0 .
$$

Here the last inequality holds true since $\phi \geq 0$ and $\hat{w}(x) \geq 0$ for $x>N_{\varepsilon}-N$.

Thus, (3.12) is proved and yields the first inequality in (3.10). The second one can be proved similarly.

There remains to check that $\alpha_{\varepsilon}^{+} \rightarrow \alpha^{+}$when $\varepsilon \rightarrow 0$. In view of (3.6), the family $\alpha_{\varepsilon}^{+}$is bounded. Let $\beta \geq 0$ be the limit of some converging subsequence $\alpha_{\varepsilon_{n}}^{+}$. Taking the limit $n \rightarrow \infty$ in (3.11) we conclude that $\beta \geq 0$ satisfies the equation

$$
\beta^{2}-c \beta+a^{+} \int_{-\infty}^{\infty} \phi(z) e^{\beta z} d z+b^{+}=0 .
$$

Therefore due to the uniqueness result in Lemma 10 we conclude that $\beta=\alpha^{+}$so that $\alpha_{\varepsilon}^{+} \rightarrow \alpha^{+}$ when $\varepsilon \rightarrow 0$. The same proof holds for $\alpha_{\varepsilon}^{-}$. This completes the proof of the lemma.

Next we aim to investigate the location as well as some geometrical properties of the essential spectrum of $L$ under suitable transformation in some exponential weighted spaces. For that purpose, let us recall that some bounded linear operator $A: E \rightarrow F$ is said to be normally solvable (or a semi-Fredholm operator) if it has a finite dimensional kernel and a closed range. A linear operator $A$ satisfies the Fredholm property if it is normally solvable and has a finite codimensional space. Then we can introduce the two following sets, the semi-Fredholm spectrum and the Fredholm spectrum:

$$
\sigma_{N S}(A)=\{\lambda \in \mathbb{C}:(A-\lambda) \text { is not normally solvable }\},
$$




$$
\sigma_{\text {ess }}(A)=\{\lambda \in \mathbb{C}: \quad A-\lambda \text { is not Fredholm }\} .
$$

Coming back to the operator $L$, by using some results proved by Apreutesei et al. in $[2,3]$, the location of the semi-Fredholm spectrum can be characterized in terms of the limiting operators $L^{ \pm}$:

Lemma 12. For each $\lambda \in \mathbb{C}$, the operator $L-\lambda$ has a closed range and a finite dimensional kernel if and only if the equations $\left(L^{ \pm}-\lambda\right) u=0$ have no bounded and nontrivial solution.

From this lemma we can derive the following useful characterization :

Lemma 13. The semi-Fredholm spectrum of operator $L: E \rightarrow F$ is given by

$$
\sigma_{N S}(L)=\left\{-\xi^{2}+i \xi c+b^{ \pm}+a^{ \pm} \int_{-\infty}^{\infty} \phi(z) e^{-i \xi z} d z ; \quad \xi \in \mathbb{R}\right\}
$$

Then we have the following link between the semi-Fredholm spectrum and the Fredholm spectrum:

Lemma 14. The following holds:

(i) $\sigma_{N S}(L) \subset \sigma_{e s s}(L)$.

(ii) Let $\lambda \in \mathbb{C}$ be given. If for any $\rho \geq 0$ the operator $L-(\lambda+\rho)$ is normally sovable then $(L-\lambda)$ satisfies the Fredholm property.

The proof of this lemma can be found in Theorem 3.2 [2] and in [3].

We will investigate the operator $L$ as acting in some exponential weighted spaces. We consider two regular functions $\theta_{1}, \theta_{2}: \mathbb{R} \rightarrow \mathbb{R}$ such that

$$
\begin{aligned}
& \theta_{1}(x)=\left\{\begin{array}{l}
x \text { if } x \geq 1 \\
0 \text { if } x \leq 0
\end{array}\right. \\
& \theta_{2}(x)=\left\{\begin{array}{l}
x \text { if } x \leq-1 \\
0 \text { if } x \geq 0
\end{array}\right.
\end{aligned}
$$

and the weighted function, for each $\tau \in \mathbb{R}$ :

$$
\omega_{\tau}(x)=e^{\tau \theta(x)}, \quad x \in \mathbb{R} \text {, with } \theta(x)=\alpha^{+} \theta_{1}(x)-\alpha^{-} \theta_{2}(x) .
$$

where $\alpha^{+}$and $\alpha^{-}$are given by (3.4). It satisfies

$$
w_{\tau}(x)=\left\{\begin{array}{l}
e^{\tau \alpha^{+} x} \text { if } x \geq 1 \\
e^{-\tau \alpha^{-} x} \text { if } x \leq-1 .
\end{array}\right.
$$

Next we introduce the operator $L_{\tau}: E \rightarrow F$ defined by

$$
L_{\tau} v=\omega_{\tau} L\left(\frac{v}{\omega_{\tau}}\right) .
$$


The explicit form of $L_{\tau}$ reads

$$
\begin{aligned}
L_{\tau} v= & v^{\prime \prime}+\left(c-2 \tau \theta^{\prime}(x)\right) v^{\prime}+\left\{b(x)-c \tau \theta^{\prime}(x)+\tau^{2} \theta^{\prime}(x)^{2}-\tau \theta^{\prime \prime}(x)\right\} v \\
& +a(x) \int_{-\infty}^{\infty} \phi(y) e^{\tau(\theta(x)-\theta(x-y))} v(x-y) d y .
\end{aligned}
$$

The essential spectrum of operator $L_{\tau}$ can easily be computed.

Lemma 15. For each $\tau \in \mathbb{R}$, the essential spectrum of the operator $L_{\tau}$ is given by

$$
\sigma_{N S}\left(L_{\tau}\right)=\left\{\Phi^{+}\left(\xi, \tau \alpha^{+}\right), \xi \in \mathbb{R}\right\} \cup\left\{\Phi^{-}\left(\xi, \tau \alpha^{-}\right), \xi \in \mathbb{R}\right\},
$$

wherein we have set

$$
\begin{aligned}
& \Phi^{+}\left(\xi, \tau \alpha^{+}\right)=-\xi^{2}+i \xi\left(c-2 \tau \alpha^{+}\right)+\left(\tau \alpha^{+}\right)^{2}-c \tau \alpha^{+}+a^{+} \int_{-\infty}^{\infty} \phi(z) e^{\tau \alpha^{+} z-i \xi z} d z+b^{+} \\
& \Phi^{-}\left(\xi, \tau \alpha^{-}\right)=-\xi^{2}+i \xi\left(c+2 \tau \alpha^{-}\right)+\left(\tau \alpha^{-}\right)^{2}+c \tau \alpha^{-}+a^{-} \int_{-\infty}^{\infty} \phi(z) e^{-\tau \alpha^{-} z-i \xi z} d z+b^{-}
\end{aligned}
$$

Moreover for each $\lambda \in \mathbb{C}$ iffor each $\rho \geq 0$ we have $\lambda+\rho \notin \sigma_{N S}\left(L_{\tau}\right)$ then $\lambda \notin \sigma_{\text {ess }}\left(L_{\tau}\right)$.

In particular recalling (3.4), we have

$$
\Phi^{ \pm}\left(0, \alpha^{ \pm}\right)=0
$$

so that $0 \in \sigma_{N S}\left(L_{1}\right)$.

We shall now prove some suitable geometrical continuation property for the essential spectrum.

Proposition 16. Under assumptions (3.2)-(3.3), the following properties hold true:

(i) For each $\tau \in[0,1)$,

$$
\sigma_{\text {ess }}\left(L_{\tau}\right) \subset\{z \in \mathbb{C}: \Re z<0\} .
$$

(ii) For each $\lambda \in\{z \in \mathbb{C}: \Re z \geq 0, \quad z \neq 0\}$ and each $\tau \in[0,1]$,

$$
0 \notin \sigma_{\text {ess }}\left(L_{\tau}-\lambda\right) \text {. }
$$

Remark 17. For each $\lambda \in\{z \in \mathbb{C}: \Re z \geq 0\}$ let the number $\tau(\lambda) \geq 0$ be defined by

$$
\tau(\lambda)=\inf \left\{\tau \geq 0: 0 \in \sigma_{e s s}\left(L_{\tau}-\lambda\right)\right\} .
$$

Then $\tau(0)=1$ while $\tau(\lambda)>1$ for each $\lambda \in\{z \in \mathbb{C}: \quad \Re z \geq 0, \quad z \neq 0\}$. Indeed the map $\tau \rightarrow L_{\tau}$ is continuous from $\mathbb{C}$ into $\mathcal{L}(E, F)$ while the set of Fredholm operators is an open subset of $\mathcal{L}(E, F)$. 
From a geometrical point of view, $(i)$ and $(i i)$ in Proposition 16 (for $\tau=0$ ) means that the essential spectrum of the operator $L$ is in the left half plane. When it is shifted according to the parameter $\tau$, that corresponds to increase the weighted function at infinity, the first imaginary point of the essential spectrum that is reached is the origin and it is obtained for $\tau=1$. It follows from (3.19) that $\lambda=0$ belongs to the essential spectrum when $\tau=1$.

In order to explain this result, let us consider the following example. If the function $\phi$ in the definition of the operator $L$ is the Dirac $\delta$-function, then the integral disappears and we obtain a second order ordinary differential operator. Its essential spectrum is given by the parabolas $\lambda^{ \pm}(\xi)=\Phi^{ \pm}(\xi, 0)$. Assume that they are in the left-half plane of the complex plane. We move these curves to the right while their vertices come to the origin. In this case we obtain the curves $\lambda^{ \pm}(\xi)=\Phi^{ \pm}\left(\xi, \tau \alpha^{ \pm}\right)$. Then, the above result follows from the convexity of the essential spectrum for "local" operators.

The proof of Proposition 16 follows from Lemma 15 together with the following result:

Lemma 18. Under assumptions (3.2)-(3.3), the following properties hold true:

(i) For each $\tau \in[0,1)$,

$$
\sigma_{N S}\left(L_{\tau}\right) \subset\{z \in \mathbb{C}: \Re z<0\} .
$$

(ii) For each $\lambda \in\{z \in \mathbb{C}: \Re z \geq 0, \quad z \neq 0\}$ and each $\tau \in[0,1]$,

$$
0 \notin \sigma_{N S}\left(L_{\tau}-\lambda\right) \text {. }
$$

Proof. Coming back to relations (3.18), for any $\tau \geq 0$ and $\xi \in \mathbb{R}$ we have

$$
\begin{aligned}
& \Re \Phi^{+}\left(\xi, \tau \alpha^{+}\right)=h^{+}\left(\tau \alpha^{+}\right)-\left(\xi^{2}+a^{+} \int_{-\infty}^{\infty} \phi(z) e^{\tau \alpha^{+} z}(1-\cos (\xi z)) d z\right), \\
& \Re \Phi^{-}\left(\xi, \tau \alpha^{-}\right)=h^{-}\left(\tau \alpha^{-}\right)-\left(\xi^{2}+a^{-} \int_{-\infty}^{\infty} \phi(z) e^{-\tau \alpha^{-} z}(1-\cos (\xi z)) d z\right),
\end{aligned}
$$

wherein the maps $h^{ \pm}$are defined by

$$
\begin{aligned}
& h^{+}(\alpha)=\alpha^{2}-c \alpha+a^{+} \int_{-\infty}^{\infty} \phi(z) e^{\alpha z} d z+b^{+} \\
& h^{-}(\beta)=\beta^{2}+c \beta+a^{-} \int_{-\infty}^{\infty} \phi(z) e^{-\beta z} d z+b^{-} .
\end{aligned}
$$

According to the proof of Lemma 10, the maps $h^{ \pm}$are convex while by (3.19) we see that $h^{ \pm}\left(\alpha^{ \pm}\right)=\Re \Phi^{+}\left(0, \alpha^{+}\right)=0$. Consequently the first term in (3.20) satisfies

$$
h^{ \pm}\left(\tau \alpha^{ \pm}\right)\left\{\begin{array}{l}
<0 \text { when } \tau \in[0,1) \\
>0 \text { when } \tau>1
\end{array}\right.
$$

Since the other term in (3.20) is negative, we conclude that

$$
\Re \Phi^{ \pm}\left(\xi, \tau \alpha^{ \pm}\right)<0 \quad \forall \xi \in \mathbb{R}, \quad \forall \tau \in[0,1),
$$


that completes the proof of $(i)$.

Let us now derive $(i i)$. Let $\lambda \in\{z \in \mathbb{C}: \Re z \geq 0, \quad z \neq 0\}$ be given. By the above inequality we have

$$
\Re\left(\Phi^{ \pm}\left(\xi, \tau \alpha^{ \pm}\right)-\lambda\right) \leq-\Re \lambda, \quad \forall \xi \in \mathbb{R}, \forall \tau \in[0,1] .
$$

This yields $(i i)$ if $\Re \lambda>0$.

Let us now assume that $\lambda=i \mu_{0}$ for some given $\mu_{0} \in \mathbb{R} \backslash\{0\}$. We claim that the equation $\Phi^{ \pm}\left(\xi, \tau \alpha^{ \pm}\right)=i \mu_{0}$ with $\xi \in \mathbb{R}$ and $\tau \in[0,1]$ has no solution. We will only investigate the equation with the + signs. The other one can be held similarly. The equation $\Phi^{+}\left(\xi, \tau \alpha^{+}\right)=i \mu_{0}$ re-writes as

$$
\begin{aligned}
& h^{+}\left(\tau \alpha^{+}\right)-\left(\xi^{2}+a^{+} \int_{-\infty}^{\infty} \phi(z) e^{\tau \alpha^{+} z}(1-\cos (\xi z)) d z\right)=0 \\
& \xi\left(c-2 \tau \alpha^{+}\right)-a^{+} \int_{-\infty}^{\infty} \phi(z) e^{\tau \alpha^{+} z} \sin (\xi z) d z=\mu_{0} .
\end{aligned}
$$

Here since $\tau \in[0,1]$ we have $h^{+}\left(\tau \alpha^{+}\right) \leq 0$. Therefore the first equation implies that $\xi=0$. Then the second equation yields $\mu_{0}=0$, whence a contradiction. This shows $(i i)$ and concludes the proof of Lemma 18.

Lemma 19. Under assumptions (3.2)-(3.3), let $\lambda \in \mathbb{C}$ with $\Re \lambda \geq 0$ and $\lambda \neq 0$ be given. Then there exists $\widehat{\tau}>1$ such that the number of linearly independent solutions of the equation

$$
\left(L_{\tau}-\lambda\right) u=0,
$$

does not depend on $\tau \in[0, \widehat{\tau}]$. Moreover if $u$ is a solution of $(L-\lambda) u=0$ then $\omega_{\widehat{\tau}} u \in E$ and

$$
\lim _{|x| \rightarrow \infty} \omega_{\widehat{\tau}}(x) u(x)=0 .
$$

The proof of this result relies on a fundamental result for Fredholm operators [10] :

Lemma 20. Let $E$ and $F$ be two Banach spaces. Let $\mathcal{O}$ be a domain in $\mathbb{C}$ and $H: \mathcal{O} \rightarrow$ $\mathcal{L}(E, F)$ be an holomorphic map. Let $G$ be a connected component of the set $\{\tau \in \mathcal{O}: H(\tau)$ is a Fredholm operator $\}$. Then the index $\kappa(H(\tau))$ is constant on $G$ while the quantities dim ker $H(\tau)$ and codim ker $H(\tau)$ are also constant on $G$ except for some possible isolated points $\tau$ where they have greater values.

Proof. We first extend the definition of the operator $L_{\tau}$ for $\tau \in \mathbb{C}$ by (3.17). Note that for any $\tau \in \mathbb{C}$ we have $L_{\tau}=L+\tau K(\tau)$ where $K(\tau): E \rightarrow F$ is a bounded linear operator defined by

$$
K(\tau) v=-2 \theta^{\prime}(x) v^{\prime}+\left\{-c \theta^{\prime}(x)+\tau \theta^{\prime}(x)^{2}-\theta^{\prime \prime}(x)\right\} v+a(x) \int_{-\infty}^{\infty} \phi(y) \frac{e^{\tau(\theta(x)-\theta(x-y))}-1}{\tau} v(x-y) d y .
$$

Then this operator can be extended into series power (converging for the topology of $\mathcal{L}(E, F)$ ) with respect to $\tau \in \mathbb{C}$ by

$$
K(\tau)=K_{0}+\sum_{n=1}^{\infty} \tau^{n} K_{n}
$$


with

$$
\begin{gathered}
K_{0} v=b(x) \int_{-\infty}^{\infty} \phi(y)(\theta(x)-\theta(x-y)) v(x-y) d y-2 \theta^{\prime}(x) v^{\prime}-\left\{c \theta^{\prime}(x)+\theta^{\prime \prime}(x)\right\} v, \\
K_{1} v=\theta^{\prime}(x)^{2} v+b(x) \int_{-\infty}^{\infty} \phi(y) \frac{(\theta(x)-\theta(x-y))^{2}}{2} v(x-y) d y \\
K_{n} v=b(x) \int_{-\infty}^{\infty} \phi(y) \frac{(\theta(x)-\theta(x-y))^{n+1}}{(n+1) !} v(x-y) d y, \quad n \geq 2 .
\end{gathered}
$$

Thus the map $H: \mathbb{C} \rightarrow \mathcal{L}(E, F)$ defined by $H(\tau)=L_{\tau}-\lambda$ is holomorphic on $\mathbb{C}$.

In view of Proposition $16(i i)$ and Remark 17, there exists $\widehat{\tau}>1$ such that $[0, \widehat{\tau}]$ is a subset of some connected component $G$ of $\left\{\tau \in \mathbb{C}:\left(L_{\tau}-\lambda\right)\right.$ is a Fredholm operator $\}$. Lemma 20 yields that the quantity $\operatorname{dim} \operatorname{ker} L_{\tau}$ is constant on $G$ except for some possible isolated points $\tau$ where it takes a greater value. Let us check that such points can not exist. Indeed arguing by contradiction assume that $\tau_{0} \in G$ is such an isolated point with $\hat{m}=\operatorname{dim} \operatorname{ker} H\left(\tau_{0}\right) \geq 1$. For $\delta>0$ small enough we have $\tau_{0}-\delta \in G$ and

$$
\operatorname{dim} \operatorname{ker} H\left(\tau_{0}-\delta\right)<\hat{m}
$$

Let $v_{1}, \ldots, v_{\hat{m}}$ in $E$ be linearly independent solutions of the equation $H\left(\tau_{0}\right) u=0$. Then the functions $\widetilde{v_{i}}=v_{i} \omega_{\delta} \in E$ are linearly independent. Furthermore since $\omega_{\tau_{0}-\delta}=\omega_{\tau_{0}} \omega_{-\delta}$ they satisfy

$$
H\left(\tau_{0}-\delta\right) \widetilde{v_{i}}=\frac{\omega_{\tau_{0}-\delta}}{\omega_{\tau_{0}}} H\left(\tau_{0}\right) v_{i}=0
$$

This contradicts (3.24) and provides that $m=\operatorname{dim} \operatorname{ker}\left(L_{\tau}-\lambda\right)$ does not depend on $\tau \in[0, \widehat{\tau}]$. Actually this proof shows that for each $\tau_{0} \in G$, the map $\delta \rightarrow \operatorname{dim} \operatorname{ker} H\left(\tau_{0}-\delta\right)$ is decreasing on the set $\left\{\delta>0: \tau_{0}-\delta \in G\right\}$.

Let $\hat{v}_{1}, \ldots, \hat{v}_{m}$ denote some basis of $\operatorname{ker}\left(L_{\widehat{\tau}}-\lambda\right)$. Then for any $i=1, . ., m$ the functions $w_{i}=$ $\hat{v}_{i} / \omega_{\widehat{\tau}} \in E$ satisfy $(L-\lambda) w_{i}=0$ and constitute a basis of $\operatorname{ker}(L-\lambda)$. Therefore any $u$ satisfying $(L-\lambda) u=0$ is a linear combination of the $w_{i}$ 's and the result follows.

Lemma 21. Under assumptions (3.2)-(3.3), let $\lambda \in \mathbb{C}$ with $\Re \lambda \geq 0$ and $\lambda \neq 0$ be given and let $u$ be a solution of $(L-\lambda) u=0$. Then

$$
|u(x)|=o\left(u_{0}(x)\right) x \rightarrow \pm \infty .
$$

Proof. We will only prove that

$$
\lim _{x \rightarrow \infty} \frac{u(x)}{u_{0}(x)}=0
$$

The proof of the analog limit as $x \rightarrow-\infty$ is similar. Recall that Lemma 11 guarantees the existence of $m_{\varepsilon}>0$ such that

$$
\liminf _{x \rightarrow \infty} u_{0}(x) e^{\alpha_{\varepsilon}^{+} x} \geq m_{\varepsilon},
$$


where $\alpha_{\varepsilon}^{+} \rightarrow \alpha^{+}$when $\varepsilon \rightarrow 0^{+}$. Next Lemma 19 enables to say that $|u(x)|=o\left(e^{-\widehat{\tau} \alpha^{+} x}\right)$ when $x \rightarrow \infty$ with $\widehat{\tau}>1$. For $\varepsilon>0$ small enough so that

$$
\alpha_{\varepsilon}^{+}<\widehat{\tau} \alpha^{+}
$$

by combining the above properties we conclude that

$$
\frac{|u(x)|}{u_{0}(x)}=o\left(e^{\left(\alpha_{\varepsilon}^{+}-\widehat{\tau} \alpha^{+}\right) x}\right) \text { when } x \rightarrow \infty .
$$

We now complete the proof of Theorem 8 .

Proof of Theorem 8. Let us argue by contradiction and assume that there exists an eigenvalue $\widehat{\lambda}=$ $\kappa+i \mu \neq 0$ of $L$ with $\kappa \geq 0$. Let $u=u^{1}+i u^{2}$ be an associated eigenvector with $u^{1}, u^{2} \in E$. We may assume that $\mu \geq 0$ and $u^{1}$ is not identically equal to zero. Indeed if $u^{1} \equiv 0$ then $\mu=0$ and $u^{2} \neq 0$ is an eigenvector associated with $\kappa>0$. More precisely without loss of generality we may assume that

$$
\exists x_{0} \in \mathbb{R} \quad u^{1}\left(x_{0}\right)<0 .
$$

Let us introduce the function $v: \mathbb{R} \times[0, \infty) \rightarrow \mathbb{R}$ defined by

$$
v(x, t)=e^{\kappa t}\left(u^{1}(x) \cos \mu t-u^{2}(x) \sin \mu t\right),
$$

that satisfies the parabolic equation

$$
\begin{aligned}
& \frac{\partial v}{\partial t}=L v, \quad t>0, x \in \mathbb{R} \\
& v(x, 0)=u^{1}(x), \quad \forall x \in \mathbb{R} .
\end{aligned}
$$

Then we introduce the set

$$
\mathcal{A}=\left\{\gamma \geq 0 ; \gamma u^{1}(x)+u_{0}(x) \geq 0, \forall x \in \mathbb{R}\right\} .
$$

This set is not empty since $0 \in \mathcal{A}$. Furthermore it is bounded since due to (3.26) for sufficiently large $\gamma$, we have $\gamma u^{1}\left(x_{0}\right)+u_{0}\left(x_{0}\right)<0$, so that $\gamma \notin \mathcal{A}$. Let us consider

$$
\gamma_{0}=\sup \mathcal{A} \in[0, \infty)
$$

we can deduce from the definition of $\gamma_{0}$ that there exists $x_{1} \in \mathbb{R}$ such that

$$
\gamma_{0} u^{1}\left(x_{1}\right)+u_{0}\left(x_{1}\right)=0 .
$$

Indeed in view of Lemma 21, we have $\left|u^{1}(x)\right|=o\left(u_{0}(x)\right)$ as $x \rightarrow \infty$ so that $\gamma u^{1}(x)+u_{0}(x)>0$ for large enough $x$ and $\gamma \in\left[\gamma_{0}, \gamma_{0}+1\right]$. Thus a straightforward contradiction argument readily yields (3.28). 
We now consider the map $w: \mathbb{R} \times[0, \infty) \rightarrow \mathbb{R}$ given by

$$
w(x, t)=\gamma_{0} v(x, t)+u_{0}(x),
$$

that satisfies

$$
\begin{aligned}
& \frac{\partial w}{\partial t}=L w, \quad t>0, \quad x \in \mathbb{R} \\
& w(x, 0)=\gamma_{0} u^{1}(x)+u_{0}(x) \geq 0, \quad x \in \mathbb{R} .
\end{aligned}
$$

Theorem 5 provides that $w(x, t) \geq 0$ for all $(x, t) \in \mathbb{R} \times[0, T]$. Furthermore either $w(x, t) \equiv 0$ for any $(x, t) \in \mathbb{R} \times[0, T]$ or $w(x, t)>0$ for any $(x, t) \in \mathbb{R} \times] 0, T]$.

Let us check that $w$ cannot have these properties. We first consider the case $\mu>0$. Then in view of definitions (3.29) and (3.27) we have

$$
w\left(x_{1}, \frac{2 \pi}{\mu}\right)=\gamma_{0} u^{1}\left(x_{1}\right) e^{\kappa \frac{2 \pi}{\mu}}+u_{0}\left(x_{1}\right) .
$$

Now if $\kappa>0$, since $u^{1}\left(x_{1}\right)<0$, (3.30) provides $w\left(x_{1}, \frac{2 \pi}{\mu}\right)<\gamma_{0} u^{1}\left(x_{1}\right)+u_{0}\left(x_{1}\right)=0$, that contradicts $w \geq 0$. If $\kappa=0$, (3.30) gives $w\left(x_{1}, \frac{2 \pi}{\mu}\right)=0$ so that $w(x, t) \equiv 0$ for any $(x, t) \in$ $\mathbb{R} \times[0, T]$. This implies $\mu=0$, whence a contradiction.

We now assume $\mu=0$ and $\kappa>0$. Then for any $t>0$ we have $w\left(x_{1}, t\right)=\gamma_{0} e^{\kappa t} u^{1}\left(x_{1}\right)+$ $u_{0}\left(x_{1}\right)<\gamma_{0} u^{1}\left(x_{1}\right)+u_{0}\left(x_{1}\right)=0$, that is impossible

Therefore the operator $L$ has no eigenvalue with non-negative real part except the zero eigenvalue.

It remains to prove that zero is a simple eigenvalue (here simple is understood in the sense of the dimension of its generalized eigenspace). We first show that ker $L=\operatorname{span} u_{0}$. Indeed let us consider the equation

$$
L u=0, u \in E .
$$

Let us consider $\hat{\gamma} \in \mathbb{R}$, the infimum value of $\gamma \in \mathbb{R}$ such that $u+\gamma u_{0} \geq 0$ on $[-N, N]$. Then the function $w=u+\widehat{\gamma} u_{0}$ satisfies the equation

$$
L w=0, w \geq 0 \text { on }[-N, N] \text { and } \exists \widehat{x} \in[-N, N] w(\widetilde{x})=0 .
$$

Therefore, thanks to Lemmas 6 and 7, we obtain that $w \geq 0$ on $\mathbb{R}$ and either $w>0$ on $\mathbb{R}$ or $w \equiv 0$. Due to the definition of $\widehat{x} \in[-N, N]$, we have $z \equiv 0$ so that $u=-\widehat{\gamma} u_{0}$ and ker $L=\operatorname{span} u_{0}$.

Let us show that ker $L^{2}=\{0\}$. Arguing by contradiction let us assume that there exists $u \in E$ such that

$$
L u=-u_{0} .
$$

Let us consider $\widetilde{\gamma} \in \mathbb{R}$, the infimum value of $\gamma \in \mathbb{R}$ such that $u+\gamma u_{0} \geq 0$ on $[-N, N]$. Then the function $z=u+\widetilde{\gamma} u_{0}$ satisfies the equation

$$
L z+u_{0}=0, \text { and } \exists \widetilde{x} \in[-N, N] z(\widetilde{x})=0 .
$$


Thus $L z=-u_{0}<0$ on $\mathbb{R}$ while $z \geq 0$ on $[-N, N]$. Therefore, thanks to Lemmas 6 and 7 , we obtain that $z \geq 0$ and either $z>0$ on $\mathbb{R}$ or $z \equiv 0$. Due to the definition of $\widetilde{x} \in[-N, N]$, we have $z \equiv 0$ so that $u=-\widetilde{\gamma} u_{0}$. Hence $L u=0$, that contradicts $L u=-u_{0}<0$. This completes the proof of the theorem.

We conclude this section by giving a corollary of Theorem 8. For any $\tau \geq 0$ we consider the Banach spaces

$$
E_{\tau}=\left\{v \in E: \omega_{\tau} v \in E\right\}, \quad F_{\tau}=\left\{v \in F: \omega_{\tau} v \in F\right\}
$$

and the operator $\widehat{L}_{\tau} v=L v$, where $L$ is defined in (3.1). The operator $\widehat{L}$ is acting from $E_{\tau}$ into $F_{\tau}$. Then we have the following result:

Corollary 22. Let assumptions (3.2)-(3.3) hold. Let us assume that there exists $\tau \in[0,1)$ and $u_{0} \in E_{\tau}$ such that

$$
u_{0}>0 \text { and } \widehat{L}_{\tau} u_{0}=0 .
$$

Then zero is a simple eigenvalue of the operator $\widehat{L}_{\tau}: E_{\tau} \rightarrow F_{\tau}$ while the remainder of its spectrum is strictly contained in the left half plane.

Proof. Let us first notice that $\widehat{L}_{\tau}: E_{\tau} \rightarrow F_{\tau}$ and $L_{\tau}$ are related to each other through (3.16). Hence the resolvent sets of these two operators coincide as well as the complementary of their essential spectrum. Due to Proposition 16, since $\tau \in[0,1)$, the essential spectrum of $\widehat{L}_{\tau}: E_{\tau} \rightarrow F_{\tau}$ is strictly contained in the left half plane. Moreover it is easily checked that any eigenvalue of the operator $\widehat{L}_{\tau}$ is also an eigenvalue of the operator $L$. Therefore Theorem 8 applies and this completes the proof of the result.

\section{Linear stability of travelling wave solutions}

The aim of this section is to study the local stability of monotonic wave solution of problem (2.1). For that purpose we shall assume that (3.3) holds, that the function $f$ is of class $C^{1}$ on $\mathbb{R}^{2}$ and that there exist two real numbers $w^{-}<w^{+}$such that

$$
f\left(w^{ \pm}, w^{ \pm}\right)=0
$$

A travelling wave solution of problem (2.1) consists in a bounded function $w_{0}: \mathbb{R} \rightarrow \mathbb{R}$ of class $C^{2}$ and a real $c \in \mathbb{R}$ the wave speed such that the function

$$
u(x, t)=w_{0}(x-c t), \quad x \in \mathbb{R}, t>0,
$$

is a solution of (2.1) together with

$$
\lim _{z \rightarrow \pm \infty} w_{0}(z)=w^{ \pm}
$$


It is easily seen that such a travelling wave solution $\left(w_{0}, c\right)$ satisfies

$$
\left\{\begin{array}{l}
w_{0}^{\prime \prime}+c w_{0}^{\prime}+f\left(w_{0}, J\left(w_{0}\right)\right)=0 \\
\lim _{z \rightarrow \pm \infty} w_{0}(z)=w^{ \pm}
\end{array}\right.
$$

Our aim is to investigate the local stability with shift of such a wave solution. We do not address the problem of existence of such a solution. This problem has been investigated for instance in $[2,3,6]$.

The stability result is related to some spectral properties of the linearized operator around the wave solution $w_{0}$, that reads

$$
L w=w^{\prime \prime}+c w^{\prime}+a(x) \phi * w+b(x) w,
$$

wherein we have set

$$
a(x)=\frac{\partial f\left(w_{0}(x), J\left(w_{0}\right)(x)\right)}{\partial v}, \quad b(x)=\frac{\partial f\left(w_{0}(x), J\left(w_{0}\right)(x)\right)}{\partial u}, \quad x \in \mathbb{R} .
$$

Let us also set

$$
a^{ \pm}=\frac{\partial f\left(w^{ \pm}, w^{ \pm}\right)}{\partial v}, \quad b^{ \pm}=\frac{\partial f\left(w^{ \pm}, w^{ \pm}\right)}{\partial u} .
$$

Let us assume that the following assumptions hold:

The maps $f: \mathbb{R}^{2} \rightarrow \mathbb{R}$ is of the class $C^{1}$,

$$
\begin{aligned}
& \frac{\partial f(u, v)}{\partial v} \geq 0, \quad \forall(u, v) \in\left[w^{-}, w^{+}\right]^{2}, \\
& \frac{\partial f\left(w^{ \pm}, w^{ \pm}\right)}{\partial u}+\frac{\partial f\left(w^{ \pm}, w^{ \pm}\right)}{\partial v}<0 .
\end{aligned}
$$

In addition, let us assume that

$$
\text { Problem (4.2) has a solution }\left(w_{0}, c\right) \text { such that } w_{0}^{\prime}(x)>0 \forall x \in \mathbb{R} \text {. }
$$

In view of definitions (4.4) and (4.5), one can notice that the last inequality in (4.6) re-writes as $a^{ \pm}+b^{ \pm}<0$.

Before stating our main result, let us introduce some notations and definitions. Let us first recall that due to Assumption (4.6) operator $L$ defined in (3.1) satisfies Assumption (3.2). Therefore one may define the quantities $\alpha^{ \pm}>0$ by using (3.19) as well as the map $\omega_{\tau}$ in (3.15) for any $\tau \in \mathbb{C}$. Together with these notations, let us introduce the Banach space $X=C_{b}(\mathbb{R}, \mathbb{R})$, the space of bounded and continuous map from $\mathbb{R}$ into $\mathbb{R}$. Moreover, for any $\tau \in \mathbb{R}$ we consider the weighted space $C_{\tau}$ defined by

$$
C_{\tau}=\left\{u \in C_{b}(\mathbb{R}): \lim _{x \rightarrow \pm \infty} \omega_{\tau}(x) u(x)=0\right\} .
$$

We shall also consider the space

$$
C_{\tau}^{2}=\left\{u \in C_{\tau} \cap C^{2}(\mathbb{R}, \mathbb{R}): u^{\prime}, u^{\prime \prime} \in C_{\tau}\right\} .
$$


The space $X$ is endowed with the usual supremum norm denoted by \|\|$_{\infty}$ while the spaces $C_{\tau}$ and $C_{\tau}^{2}$ are endowed with the weighted norms

$$
\|u\|_{\tau}=\left\|\omega_{\tau}(.) u\right\|_{\infty}, \quad \forall u \in C_{\tau}, \quad\|u\|_{2, \tau}=\|u\|_{\tau}+\left\|u^{\prime}\right\|_{\tau}+\left\|u^{\prime \prime}\right\|_{\tau}, \quad \forall u \in C_{\tau}^{2} .
$$

Then we have the following definition (see [18])

Definition 23. (Asymptotic stability with shift) Let $\left(Y,\|\|_{Y}\right)$ be a Banach space contained in $X$. Let $\left(w_{0}, c\right) \in X \times \mathbb{R}$ be a solution of (4.2). Then $\left(w_{0}, c\right)$ is said to be asymptotically stable with shift with respect to $\left(Y,\|\|_{Y}\right)$ if there exists $\varepsilon>0$ such that for any $u_{0} \in X$ such that

$$
u_{0}-w_{0} \in Y \text { and }\left\|u_{0}-w_{0}\right\|_{Y}<\varepsilon
$$

then the solution of the evolution problem

$$
\left\{\begin{array}{l}
\frac{\partial u}{\partial t}=\frac{\partial^{2} u}{\partial x^{2}}+c \frac{\partial u}{\partial x}+f(u, J(u)), x \in \mathbb{R}, t>0 \\
u(., 0)=u_{0}
\end{array}\right.
$$

exists for all $t>0$, is unique and satisfies that

$$
\begin{aligned}
& u(., t)-w_{0} \in Y \quad \forall t>0 \\
& \left\|u(., t)-w_{0}(.+h)\right\|_{Y} \leq M e^{-b t} \forall t>0
\end{aligned}
$$

where $h \in \mathbb{R}$ depends on $u_{0}, M>0$ and $b>0$ are independent of $t>0, u_{0}$ and $h$.

We are now able to state our main result:

Theorem 24. Let Assumptions (4.6) and (4.7) be satisfied. Let us assume that $w_{0}^{\prime} \in C_{\tau}$ for some $\tau \in[0,1)$. Then $\left(w_{0}, c\right)$ is asymptotically stable with shift with respect to the space $\left(C_{\tau},\|\|_{\tau}\right)$.

If we recall definition (4.8), we obtain that

$$
C_{0}=\left\{u \in C_{b}(\mathbb{R}): \lim _{x \rightarrow \pm \infty} u(x)=0\right\} .
$$

Therefore Theorem 24 re-writes as follows with $\tau=0$.

Corollary 25. Let Assumptions (4.6) and (4.7) be satisfied. Let us assume that

$$
\lim _{x \rightarrow \pm \infty} w_{0}^{\prime}(x)=0
$$

Then $\left(w_{0}, c\right)$ is asymptotically stable with shift with respect to the space $\left(C_{0},\|\|_{0}\right)$.

The proof of this result relies on the application of a general result that will be exposed in the next subsection, while the proof of Theorem 24 will be given in Section 4.2. 


\subsection{A general stability result}

In this section, for the sake of completeness, we recall a general result concerning stability of a one dimensional manifold of equilibria for an abstract Cauchy problem. For that purpose let us consider a Banach space $(X,\|\|)$, a linear operator $A: D(A) \subset X \rightarrow X$ and a continuous map $h: X \rightarrow X$. Then we can consider the following Cauchy problem

$$
\left\{\begin{aligned}
\frac{d u(t)}{d t} & =A u(t)+h(u(t)), \quad t>0 \\
u(0) & =u_{0} \in X .
\end{aligned}\right.
$$

Then let us assume that the corresponding stationary equation

$$
A u+h(u)=0, \quad u \in D(A),
$$

has a one-parameter family of solutions $\varphi_{\alpha} \in D(A)$ for any $\alpha \in I$ where $I$ is some bounded interval, $I \subset \mathbb{R}$.

The aim of this section is to recall some local stability result for these family of equilibria with respect to small perturbations in some Banach space. For that purpose we introduce $(Y,\|\|)$ a Banach space such that $Y \subset X$. Then let us introduce some assumptions upon the family of equilibria $\left\{\varphi_{\alpha}\right\}_{\alpha \in I}$ as well as upon $A$ and $h$ in (4.11).

\section{- Assumption (H1)}

- (a) The map $\alpha \in I \rightarrow \varphi_{\alpha} \in X$ is differentiable and $\varphi_{\alpha}^{\prime} \in Y$ for any $\alpha \in I$.

- (b) The map $\alpha \in I \rightarrow \varphi_{\alpha}^{\prime} \in Y$ is Lipschitz continuous from $I$ into $\left(Y,\|\|_{Y}\right)$.

\section{- Assumption (H2)}

- (a) The map $h: X \rightarrow X$ is of the class $C^{1}$ on $X$.

- (b) For any $\alpha \in I$ and any $v \in Y$ the map $h^{\prime}\left(\varphi_{\alpha}^{\prime}+v\right)$ belongs to $\mathcal{L}(Y)$, the space of bounded linear operators from $Y$ into itself. Moreover for each $\alpha \in I$ the map $v \in$ $Y \rightarrow h^{\prime}\left(\varphi_{\alpha}^{\prime}+v\right) \in \mathcal{L}(Y)$ is Lipschitz continuous on $B_{Y}(0,1)=\left\{v \in Y:\|v\|_{Y} \leq 1\right\}$.

\section{- Assumption (H3)}

- (a) The part of $A$ in $Y$ denoted by $A_{Y}$ and defined by

$$
\begin{aligned}
& D\left(A_{Y}\right)=\{u \in D(A) \cap Y: A u \in Y\} \\
& A_{Y} u=A u \quad \forall u \in D\left(A_{Y}\right),
\end{aligned}
$$

is the generator of an analytic semigroup on $Y$. It follows from Assumption (H2) (b) that the operator $L: D\left(A_{Y}\right) \subset Y \rightarrow Y$ defined by

$$
L u=A_{Y} u+h^{\prime}\left(\varphi_{0}\right) u, \quad \forall u \in D\left(A_{Y}\right),
$$

is also the generator of an analytic semigroup on $Y$. 
- (b) The spectrum of operator $L$ has the following structure: zero is a simple eigenvalue while all the remainder of the spectrum is contained in a closed angle lying in the left complex half plane. This condition re-writes as there exists $a_{1}>0$ and $b_{1}>0$ such that

$$
\Re \lambda+a_{1}|\Im \lambda|+b_{1} \leq 0 \quad \forall \lambda \in \sigma(L) \backslash\{0\} .
$$

Then we have the following result:

Theorem 26. Let assumptions (H1)-(H3) be satisfied. Then there exists $\varepsilon>0$, such that for any $u_{0} \in X$ satisfying

$$
u_{0}-\varphi_{0} \in Y \text { and }\left\|u_{0}-\varphi_{0}\right\|_{Y}<\varepsilon,
$$

the abstract Cauchy problem (4.11) has a unique and globally defined solution $t \rightarrow u(t) \in X$ that satisfies

$$
\left\|u(t)-\varphi_{\alpha}\right\|_{Y} \leq M e^{-b t} \forall t>0,
$$

for some $\alpha \in I$ depending on $u_{0}$, while the constant $M>0$ and $b>0$ are independent of $u_{0}, \alpha$ and $t>0$.

The proof of this result can be found in the monograph of Volpert et al [18].

\subsection{Proof of Theorem 24}

Let us consider the linear operator $A: D(A) \subset X \rightarrow X$ defined by

$$
\begin{aligned}
& D(A)=\left\{u \in X: u^{\prime \prime}, u^{\prime} \in X\right\}, \\
& A u=u^{\prime \prime}+c u^{\prime},
\end{aligned}
$$

as well as the map $h: X \rightarrow X$ defined by

$$
h(u)(x)=f\left(u(x), \int_{-\infty}^{\infty} \phi(x-y) u(y) d y\right) .
$$

We also denote $\varphi_{\alpha}=w_{0}(.+\alpha) \in X$ for any $\alpha \in \mathbb{R}$. Let us introduce $\tau \in[0,1)$ such that $w_{0}^{\prime} \in C_{\tau}$ and let us set $Y=C_{\tau}$ endowed with the norm \|\|$_{\tau}$. Then since $w_{0}^{\prime} \in C_{\tau}$ and $\phi$ is compactly supported, we can easily check that $\alpha \rightarrow \varphi_{\alpha}$ satisfies Assumption (H1) while the map $h: X \rightarrow X$ satisfies Assumption (H2). It remains to check the operator $A$ satisfies Assumption (H3). To do that, let us first introduce the operator $A_{Y}$ defined by

$$
\begin{aligned}
& D\left(A_{Y}\right)=C_{\tau}^{2} \\
& A_{Y} u=u^{\prime \prime}+c u^{\prime} .
\end{aligned}
$$

Then we have the following lemma:

Lemma 27. The linear operator $A_{Y}: C_{\tau}^{2} \subset C_{\tau} \rightarrow C_{\tau}$ is the infinitesimal generator of an analytic semigroup. 
Proof. Let us first notice that $C_{\tau}^{2}$ is dense in $\left(C_{\tau},\|\|_{\tau}\right)$. The proof of this result is similar to the one given in the proof of Lemma 2.1 Chapter 5 in [18]. To complete the proof we shall show that $L$ is a sectorial operator on $C_{\tau}$ (see for instance $[13,16]$ for details about sectorial operators and their links together with analytic semigroups). To do that let us introduce the operator

$$
T: C_{\tau} \rightarrow C_{0}, \quad T v=\omega_{\tau} v .
$$

Note that $T$ is a bounded linear operator that is invertible and such that $T$ maps $C_{\tau}^{2}$ onto $C_{0}^{2}$. Moreover we have $T^{-1}: C_{0} \rightarrow C_{\tau}$ with $T^{-1} u=u / \omega_{\tau}$. Next we introduce the operator $\widetilde{A}: C_{0}^{2} \rightarrow$ $C_{0}$ defined by $\widetilde{A}=T A_{Y} T^{-1}$. Moreover operator $\widetilde{A}$ reads as

$$
\widetilde{A} v=v^{\prime \prime}+\left(c-2 \tau \theta^{\prime}(x)\right) v^{\prime}+\left\{\tau^{2} \theta^{\prime}(x)^{2}-c \tau \theta^{\prime}(x)-\tau \theta^{\prime \prime}(x)\right\} v .
$$

It is clear that $\rho\left(A_{Y}\right)=\rho(\widetilde{A})$ and that

$$
\left(\lambda-A_{Y}\right)^{-1}=T^{-1}(\lambda-\widetilde{A})^{-1} T, \quad \forall \lambda \in \rho\left(A_{Y}\right) .
$$

To complete the proof it remains to show that $\widetilde{A}$ is a sectorial operator on $C_{0}$. To do so let us recall that the operator $\Delta: C_{0}^{2} \rightarrow C_{0}$ defined by $\Delta \varphi=\varphi^{\prime \prime}$ is a sectorial operator on $C_{0}$ (see for instance [12]). Moreover the operator $B: D(B) \subset C_{0} \rightarrow C_{0}$ defined by $D(B)=\left\{\varphi \in C_{0}: \quad \varphi^{\prime} \in C_{0}\right\}$ and

$$
B \varphi=\left(c-2 \tau \theta^{\prime}(x)\right) \varphi^{\prime}+\left\{\tau^{2} \theta^{\prime}(x)^{2}-c \tau \theta^{\prime}(x)-\tau \theta^{\prime \prime}(x)\right\} \varphi,
$$

is a closed linear operator while for any $\gamma \in(1 / 2,1) D\left(\Delta^{\gamma}\right) \subset D(B)$. Thus by using Corollary 2.4 page 81 in Pazy [16] we get that $\widehat{A}=\Delta+B$ is a sectorial operator and the result follows.

It remains to give some information about the location of the spectrum of the operator $L$ : $C_{\tau}^{2} \rightarrow C_{\tau}$ defined in (4.3). Now since problem (4.2) is invariant with respect to translation, the map $w_{0}^{\prime} \in C_{\tau} \subset C_{0}$ is an eigenvalue of operator $L$ associated to the eigenvalue zero. Moreover since $w_{0}$ is supposed to be monotonic, we have $w_{0}^{\prime}>0$. Now due to Assumption (4.6), Corollary 22 applies and shows that zero corresponds to the principle eigenvalue of the operator $L$ and it is simple. Moreover, as explained in the proof of Corollary 22, its essential spectrum coincides with the one of operator $L_{\tau}$ (see definition (3.17).) Now due to Assumption (4.6), Proposition 16 applies. Since $\tau \in[0,1)$ it provides that $\sigma_{\text {ess }}(\widehat{L}) \subset\{z \in \mathbb{C}: \quad \Re z<0\}$. Due to the parabolic asymptotic shape of the curves $\Phi^{ \pm}\left(\xi, \tau \alpha^{ \pm}\right)$, one may include these curves into a suitable angle strictly contained in the left half plane. This means that there exist $a_{1}>0$ and $b_{1}$ such that

$$
\sigma_{N S}(\widehat{L}) \subset \mathcal{A}:=\left\{\lambda \in \mathbb{C}: \Re \lambda+a_{1}|\Im \lambda|+b_{1} \leq 0\right\} .
$$

In addition, due to Lemma 15 one can derive that $\sigma_{\text {ess }}((\widehat{L}) \subset \mathcal{A}$. Indeed for each $\lambda \in \mathbb{C}$ such that $\Re \lambda+a_{1}+b_{1}|\Im \lambda|>0$ we have

$$
\Re \lambda+\rho+a_{1}|\Im \lambda|+b_{1}>0, \quad \forall \rho \geq 0 .
$$

Therefore $\lambda+\rho \notin \sigma_{N S}(\widehat{L})$ and $\lambda \notin \sigma_{\text {ess }}(\widehat{L})$. In addition, outside of this angle there is only a finite number of eigenvalues. Thus there exists an angle strictly contained in the left half plane containing the whole spectrum of $L$ except zero which is a simple eigenvalue. This prove that Assumption (H3) holds true and complete the proof of Theorem 24 by applying Theorem 26. 


\section{Additional results and minimax representation of the wave speed}

The aim of this section is to give some additional results that are a consequence of Theorem 24 as well as the comparison principle given in Theorem 4. The details of the proofs will be omitted. They directly follow the same steps as the one of Theorem 6.1 p. 250 and Theorem 7.1 page 255 in [18]. The first result is concerned with the asymptotic behaviour of the Cauchy problem

$$
\left\{\begin{array}{l}
\frac{\partial u}{\partial t}=\frac{\partial^{2} u}{\partial x^{2}}+c \frac{\partial u}{\partial x}+f(u, J(u)), x \in \mathbb{R}, t>0, \\
u(., 0)=u_{0}
\end{array}\right.
$$

with monotonic initial condition. Recalling definitions (3.15), (4.8) and (4.10), the following result holds:

Theorem 28. Let Assumptions (4.6) and (4.7) be satisfied and assume that $w_{0}^{\prime} \in C_{\tau}$ for some $\tau \in[0,1)$. Let us assume that $u_{0} \in C_{b}(\mathbb{R})$ is some given monotonic function such that

$$
\lim _{x \rightarrow \pm \infty}\left|u_{0}(x)-w^{ \pm}\right| \omega_{\tau}(x)=0 .
$$

Then the solution $u(x, t)$ of (5.1) is globally defined and converges (with shift) to $w_{0}$ in the $C_{\tau}$-norm, that is

$$
\lim _{t \rightarrow \infty}\left\|u(., t)-w_{0}\left(.+x_{0}\right)\right\|_{\tau}=0
$$

wherein $x_{0} \in \mathbb{R}$ depends on $u_{0}$.

Next one can derive some minimax formula for the wave speed. To do so let us introduce for each $\tau \in \mathbb{R}$, the set $K_{\tau}$ defined by

$$
K_{\tau}=\left\{\rho \in C_{b}^{2}(\mathbb{R}): \quad \rho^{\prime}>0 \text { and } \lim _{x \rightarrow \pm \infty}\left|\rho(x)-w^{ \pm}\right| \omega_{\tau}(x)=0\right\} .
$$

Moreover let us set the map $\Psi: K_{\tau} \rightarrow C(\mathbb{R}, \mathbb{R})$ defined by

$$
\Psi(\rho)(x)=-\frac{\rho^{\prime \prime}(x)+f(\rho(x), \phi * \rho(x))}{\rho^{\prime}(x)}, \forall x \in \mathbb{R} .
$$

Then the following holds

Theorem 29. Let Assumptions (4.6) and (4.7) be satisfied. Let us assume that $w_{0}^{\prime} \in C_{\tau}$ for some $\tau \in[0,1)$. Then the wave speed $c$ has the following representation:

$$
c=\inf _{\rho \in K_{\tau}} \sup _{x \in \mathbb{R}} \Psi(\rho)(x)=\sup _{\rho \in K_{\tau}} \inf _{x \in \mathbb{R}} \Psi(\rho)(x) .
$$




\section{Some applications in biology and chemical kinetics}

\subsection{Population dynamics with nonlocal stimulation of reproduction}

As an application of the results given in this paper we consider the equation

$$
\frac{\partial u}{\partial t}=\frac{\partial^{2} u}{\partial x^{2}}+f(u, J(u)), t>0, \quad x \in \mathbb{R},
$$

wherein

$$
f(u, v)=k u v(1-u)-\mu u, \quad J(u)=\int_{-\infty}^{\infty} \phi(x-y) u(y, t) d y .
$$

When $\phi=\delta$ is a dirac delta function, then we obtain the local reaction-diffusion equation

$$
\frac{\partial u}{\partial t}=\frac{\partial^{2} u}{\partial x^{2}}+k u^{2}(1-u)-\mu u,
$$

where $k u^{2}(1-u)$ describes bi-sexual reproduction of a population, $u$ is the density of the population, $(1-u)$ corresponds to available resources, $\mu u$ is mortality.

In order to explain the appearance of the integral term in the equation, consider the example of sexual plant reproduction with male and female plants. We assume that they have the same density $u$ due to the same proportion at birth and to the same diffusion. Since male plants distribute their pollen in some area around their location, then $u^{2}$ in the equation should be replaced by $u$ (density of female plants) times the integral (density of pollen).

Similar models can describe evolution of cell populations where cells can produce mitosis factors diffusing in the extra-cellular matrix and stimulating proliferation of surrounding cells. The existence of travelling waves for such integro-differential equations is proved in [2], [3] in the case of functions $\phi$ with a small support and in [6] for an arbitrary support.

\subsection{Nonlocal problems in chemical kinetics}

Distribution of concentrations in a chemically reacting medium can be described by the reactiondiffusion system of equations

$$
\frac{\partial A}{\partial t}=d \frac{\partial^{2} A}{\partial x^{2}}+W(A)
$$

where $A=\left(A_{1}, \ldots, A_{m}\right)$ is the vector of concentrations, $W=\left(W_{1}, \ldots, W_{m}\right)$ is the vector of reaction rates, $d$ is the diffusion matrix. Suppose that some of the species have fast reaction and fast diffusion, that is instead of the previous systems we can write

$$
\begin{gathered}
\frac{\partial A_{i}}{\partial t}=d_{i} \frac{\partial^{2} A_{i}}{\partial x^{2}}+W_{i}(A), \quad i=1, \ldots, k, \\
\epsilon \frac{\partial A_{i}}{\partial t}=d_{i} \frac{\partial^{2} A_{i}}{\partial x^{2}}+W_{i}(A), \quad i=k+1, \ldots, m,
\end{gathered}
$$


where $\epsilon$ is a small parameter. Taking a formal limit as $\epsilon \rightarrow 0$, we obtain the equation

$$
A_{i}^{\prime \prime}+W_{i}(A)=0, \quad i=k+1, \ldots, m .
$$

We can express the last variables $A_{i}, i=k+1, \ldots, m$. This will give us integro-differential equations for the first variables $A_{i}, i=1, \ldots, k$.

One of possible examples is given by the following systems written for travelling waves:

$$
B^{\prime \prime}+c B^{\prime}+B C(1-B)-\mu B=0, \quad C^{\prime \prime}-\nu C+k B=0 .
$$

We can express $C$ from the second equation and substitute in the first equation.

\section{References}

[1] N. Apreutesei, N. Bessonov, V. Volpert and V. Vougalter, Spatial structures and generalized travelling waves for an integro-differential equation, preprint.

[2] N. Apreutesei, A. Ducrot and V. Volpert, Travelling Waves for Integro-differential Equations, D.C.D.S. B., 3 (2009), 541-561.

[3] N. Apreutesei, A. Ducrot and V. Volpert, Competition of species with intra-specific competition, Math. Modelling of Natural Phenomena, 3 (2008), 1-27.

[4] H. Berestycki, G. Nadin, B. Perthame and L. Ryzkik, The non-local Fisher-KPP equation: traveling waves and steady states, preprint.

[5] X. Chen. Existence, uniqueness, and asymptotic stability of traveling waves in nonlocal evolution equations. Adv. Differential Equations 2 (1997), no. 1, 125-160.

[6] I. Demin, V. Volpert. Existence of waves for a nonlocal reaction-diffusion equation. Math. Model. Nat. Phenom., 2010, to appear.

[7] P.C. Fife and J.B. McLeaod, The approach of solutions of nonlinear diffusion equations to traveling wave solutions, Arch. Rational Mech. Anal. 65 (1977), 355-361.

[8] A. Friedman. Partial differential equations of parabolic type. Prentice-Hall, Englewood Cliffs, 1964.

[9] S. Genieys, V. Volpert and P. Auger, Pattern and waves for a model in population dynamics with nonlocal consumption of resources, Mathematical Modelling of Natural Phenomena, 1 (2006), 65-82.

[10] I. Gohberg and M. Krein, The basic propositions on defect numbers and indices of linear operators, Amer. Math. Soc. Trans. 13, No. 2, 185-264. 
[11] S.A. Gourley, Travelling front solutions of a nonlocal Fisher equation, J. Math. Biol., 41 (2000), 272-284.

[12] D. Henry, Geometric Theory of Semilinear Parabolic Equations, Lecture Notes in Math., Vol. 840, Springer-Verlag, New York, 1981.

[13] A. Lunardi, Analytic semigroups and optimal regularity in parabolic problems, Birkhauser, Basel, 1995.

[14] S. Ma, Traveling wavefronts for delayed reaction-diffusion systems via a fixed point theorem, J. Diff. Eqs., 171 (2001), 294-314.

[15] S. Ma, Traveling waves for non-local delayed diffusion equations via auxiliary equations, J. Diff. Eqs., 237 (2007), 259-277.

[16] A. Pazy, Semigroups of operator and application to partial differential equation, SpringerVerlag, Berlin, 1983.

[17] A.I. Volpert and V.A. Volpert, Spectrum of elliptic operators and stability of travelling waves, Asymptotic Analysis 23 (2000) 111-134.

[18] A.I. Volpert, V.A. Volpert and Vl. Volpert, Travelling wave solutions of parabolic systems, Translation of Mathematical Monographs, Vol. 140, Amer. Math. Society, Providence, 1994.

[19] A. Volpert, V. Volpert. Normal solvability of general linear elliptic problems, Abstract and Applied Analysis, (2005), no. 7, 733-756.

[20] A. Volpert, V. Volpert. Fredholm property of elliptic operators in unbounded domains. Trans. Moscow Math. Soc. (2006) 67, 127-197. 